\title{
COMMENT
}

\section{Interleukin-22 from bench to bedside: a promising drug for epithelial repair}

\begin{abstract}
Cellular \& Molecular Immunology (2019) 16:666-667; https://doi.org/10.1038/s41423-018-0055-6
\end{abstract}

Cellular and Molecular Immunology (CMI), the official journal of the Chinese Society of Immunology, was established in 2004. CMI has now become one of the leading international journals in the field and publishes high-quality original articles and reviews. The very first research article published in the inaugural issue of $C M I$ was from our group and described, for the first time, the protective effect of interleukin-22 (IL-22) on the epithelium in the liver. ${ }^{1}$ Fascinatingly, 14 years later, the first-in-human phase I clinical study of human IL-22-Fc dimer (F-652) in healthy subjects is also published in $C M I^{2}$ We are very excited to see these interesting studies of IL-22 from bench to bedside published in CMI alongside its strong growth as a journal over the last 14 years.

IL-22 was first cloned in 2000 and belongs to the IL-10 family; however, IL-22 does not have any anti-inflammatory functions as IL10 does. ${ }^{3,4}$ In 2004, we reported the hepatoprotective effect of IL-22 in vivo in several mouse models of liver injury in the February issue of $\mathrm{CMI}^{1}$ and also published an immediate follow-up paper describing the underlying mechanisms by which IL-22 protects against hepatocyte injury in the May issues of Hepatology. 5 Later, studies from our laboratory and other laboratories reported that IL22 also protects against epithelial cell injury in many other organs in addition to the liver, including the pancreas, gut, kidneys, and lungs. ${ }^{6,7}$ IL-22 also plays an important role in host defense against bacterial infection. 6,7 The action of IL-22 is mainly mediated by binding to IL-10R2 and IL-22R1, and subsequently activating signal transducer and transcription factor 3 (STAT3, a survival signal for epithelial cells). ${ }^{6,7}$ IL-10R2 is ubiquitously expressed in a wide variety of cell types, whereas IL-22R1 expression is mainly restricted to epithelial cells and some fibroblasts, but is not expressed in immune cells. Therefore, IL-22 specifically targets epithelial cells and does not target immune cells, which suggests that IL-22 treatment may have minimal side effects. Indeed, IL-22 transgenic mice under the control of the albumin promoter, with high levels of serum IL-22 ( $600 \mathrm{pg} /$ $\mathrm{ml}$ ), appear normal without obvious adverse phenotypes and inflammation, and transgenic mice with super-high levels of serum IL-22 $(\sim 6000 \mathrm{pg} / \mathrm{ml})$ also appear normal, with the exception of slower body weight gain after 4 months of age, a slightly thicker epidermis and minor inflammation of the skin compared to wildtype littermate control mice. ${ }^{8}$ Interestingly, Wolk et al. ${ }^{9}$ also developed IL-22 transgenic mice, which are under the control of the lymphocyte-specific protein tyrosine kinase $(\mathrm{E} \mu \mathrm{LCK})$ promoter or the rat insulin II promoter, but most of these mice died within the first few days after birth, with one line that had severe adverse phenotypes surviving. Both the E $\mu \mathrm{LCK}$ promoter and the insulin II promoter drive gene expression during embryonic development, while the albumin promoter only induces strong gene expression after birth. This suggests that high levels of IL-22 are detrimental during embryonic development, but have minor adverse phenotypes after birth. Collectively, all of the published data from experimental models suggest that IL-22 is a promising drug for the treatment of organ failure, and its unlikeliness to generate side effects has brought several pharmaceutic companies to actively perform preclinical development of recombinant human IL-22 protein for the treatment of organ failure (unpublished data from Generon Corporation, Ltd. and a reference ${ }^{10}$ from Genentech, Inc.).

In this issue of CMI, Tang et al. ${ }^{2}$ report a first-in-human phase I clinical study of human IL-22-Fc dimer (F-652) in healthy subjects. The half-life of recombinant human IL-22 protein is less than $2 \mathrm{~h}$ in animals, which limits its clinical application. Thus, Tang et al. ${ }^{2}$ developed F-652, a recombinant fusion protein consisting of two human IL-22 molecules linked to the human IgG2 constant region $\left(\mathrm{IgG}_{2}-\mathrm{Fc}\right)$, which had $T_{1 / 2}$ values ranging from 39.4 to $206 \mathrm{~h}$ in cohorts treated with different doses of F-652. They first performed single-dose subcutaneous (SC) injection of F-652 into healthy subjects and found that some of the subjects experienced delayed injection site reactions. Thus, they moved from SC injection to intravenous (IV) injection, and found that IV dosing was better tolerated than SC dosing. No severe adverse events were observed, with the exception of mild-to-moderate skin reactions occurring at high IV doses of F-652 (30 and $45 \mu \mathrm{g} / \mathrm{kg}$ body weight). Skin reaction symptoms were well-controlled after treatment with a local steroid cream. The skin reaction after F-652 injection is probably due to IL-22-mediated activation of STAT3 and the subsequent induction of acanthosis and dermal inflammation. ${ }^{11}$

In general, IL-22 protects against epithelial cell injury and reduces bacterial infection, thereby inhibiting inflammation. ${ }^{6,7,12}$ However, IL22 has also been implicated in promoting inflammation under certain conditions, ${ }^{12}$ which raises some concerns about IL-22 therapy. The study from Tang et al. $^{2}$ revealed that IV injection of F-652 induced dose-dependent increases in serum acute-phase proteins, including serum amyloid $A$ and $C$-reactive proteins, but did not alter serum levels of a large number of pro-inflammatory cytokines/chemokines even at the highest dose of F-652 (45 $\mu \mathrm{g} / \mathrm{kg})$. These data are also consistent with the phenotypes we observed in our IL-22 transgenic mice, in which serum IL-22 levels are highly elevated (approximately $600-6000 \mathrm{pg} / \mathrm{ml}$ ) and acute-phase proteins are also elevated, but pro-inflammatory cytokines are not increased. ${ }^{8}$ Although the study from Tang et al. demonstrated that IL-22 treatment is well-tolerated and does not cause inflammation in healthy subjects, whether this also applies to patients with inflammatory diseases remains unclear and requires further evaluation. Currently, an open-label, cohort dose-escalation study to assess the safety and efficacy of F-652 is being tested in patients with severe alcoholic hepatitis. This trial is being led by the Mayo Clinic and supported by the National Institute on Alcohol Abuse and Alcoholism, National Institutes of Health, and Generon, Inc. (Shanghai). Severe alcoholic hepatitis is associated with high shortterm mortality due to hepatocellular damage, poor liver regeneration, bacterial infection, and complications. ${ }^{13}$ There are currently no Food and Drug Administration-approved drugs to effectively treat this severe disease. ${ }^{13}$ Hopefully, IL-22 in combination with other drugs can save some of these patients in the future. Another phase Ilb study of human recombinant IL-22-Fc fusion protein (F-652) is ongoing for the treatment of newly diagnosed grade II-IV lower 
gastrointestinal acute graft-vs.-host disease in hematopoietic stem cell transplantation recipients. The study is based on the protective effect of IL-22 against epithelial injury in the gastrointestinal tract and is being led by the Memorial Sloan Kettering Cancer Center. IL22 protein may also have therapeutic potential for epithelial injury in many other organs, including pancreatitis, kidney failure, and wounded skin; the effect of IL-22 on these disorders requires further evaluation.

In summary, the first-in-human phase I clinical study of human IL-22-Fc dimer in healthy subjects published by Tang et al. ${ }^{2}$ in this issue of $C M I$ shows promising results with minimal side effects in healthy subjects. Currently, a phase Ila trial is being conducted to test whether patients with severe alcoholic hepatitis also tolerate IL-22-Fc therapy, and a phase IIb trial of IL-22 treatment for severe alcoholic hepatitis has been proposed. We are looking forward to seeing these clinical trial results and hope that IL-22 therapy will be beneficial for some patients with severe alcoholic hepatitis.

\section{ACKNOWLEDGEMENTS}

This work was supported by the intramural program of the NIAAA and NIH (B.G.).

\section{ADDITIONAL INFORMATION}

Competing interests: The authors declare no competing interests.

Publisher's note: Springer Nature remains neutral with regard to jurisdictional claims in published maps and institutional affiliations.

Bin Gao ${ }^{1}$ and Xiaogang Xiang

${ }^{1}$ Laboratory of Liver Diseases, National Institute on Alcohol Abuse and Alcoholism, National Institutes of Health, Bethesda, MD 20892,

Correspondence: Bin Gao (bgao@mail.nih.gov)

\section{REFERENCES}

1. Pan, H., Hong, F., Radaeva, S. \& Gao, B. Hydrodynamic gene delivery of interleukin-22 protects the mouse liver from concanavalin $A_{-}$-, carbon tetrachloride-, and Fas ligand-induced injury via activation of STAT3. Cell. Mol. Immunol. 1, 43-49 (2004).

2. Tang K. Y., et al. Safety, pharmacokinetics, and biomarkers of F-652, a recombinant human interleukin-22 dimer, in healthy subjects. Cell Mol Immunol $2018 \mathrm{Apr}$ 18. doi: 10.1038/s41423-018-0029-8.

3. Xie, M. H. et al. Interleukin (IL)-22, a novel human cytokine that signals through the interferon receptor-related proteins CRF2-4 and IL-22R. J. Biol. Chem. 275, 31335-31339 (2000).

4. Dumoutier, L., Van Roost, E., Ameye, G., Michaux, L. \& Renauld, J. C. IL-TIF/IL-22 genomic organization and mapping of the human and mouse genes. Genes Immun. 1, 488-494 (2000).

5. Radaeva, S., Sun, R., Pan, H. N., Hong, F. \& Gao, B. Interleukin-22 (IL-22) plays a protective role in T cell-mediated murine hepatitis: IL-22 is a survival factor for hepatocytes via STAT3 activation. Hepatology 39, 1332-1342 (2004).

6. Sabat, R., Ouyang, W. \& Wolk, K. Therapeutic opportunities of the IL-22-IL-22R1 system. Nat. Rev. Drug. Discov. 13, 21-38 (2014).

7. Dudakov, J. A., Hanash, A. M. \& van den Brink, M. R. Interleukin-22: immunobiology and pathology. Annu. Rev. Immunol. 33, 747-785 (2015).

8. Park, O. et al. In vivo consequences of liver-specific interleukin-22 expression in mice: implications for human liver disease progression. Hepatology 54, 252-261 (2011).

9. Wolk, K. et al. IL-22 and IL-20 are key mediators of the epidermal alterations in psoriasis while IL-17 and IFN-gamma are not. J. Mol. Med (Berl.) 87, 523-536 (2009).

10. Stefanich, E. G. et al. Preclinical and translational pharmacology of a human interleukin-22 lgG fusion protein for potential treatment of infectious or inflammatory diseases. Biochem. Pharmacol. 152, 224-235 (2018).

11. Zheng, Y. et al. Interleukin-22, a T(H)17 cytokine, mediates IL-23-induced dermal inflammation and acanthosis. Nature 445, 648-651 (2007).

12. Alabbas, S. Y., Begun, J., Florin, T. H. \& Oancea, I. The role of IL-22 in the resolution of sterile and nonsterile inflammation. Clin. Transl. Immunol. 7, e1017 (2018).

13. Crabb, D. W. et al. Standard definitions and common data elements for clinical trials in patients with alcoholic hepatitis: recommendation from the niaaa alcoholic hepatitis consortia. Gastroenterology 150, 785-790 (2016). 